\title{
Forster, Edgar
}

\section{Männerforschung, Gender Studies und Patriarchatskritik}

Casale, Rita [Hrsg.]; Rendtorff, Barbara [Hrsg.]; Andresen, Sabine [Hrsg.]; Moser, Vera [Hrsg.]; Prengel, Annedore [Hrsg.]: Geschlechterforschung in der Kritik. Opladen ; Bloomfield Hills : Verlag Barbara Budrich 2005, S. 41-72. - (Jahrbuch Frauen- und Geschlechterforschung in der Erziehungswissenschaft; 1)

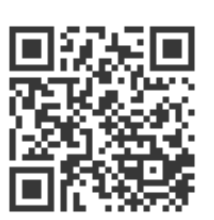

Quellenangabe/ Reference:

Forster, Edgar: Männerforschung, Gender Studies und Patriarchatskritik - In: Casale, Rita [Hrsg.];

Rendtorff, Barbara [Hrsg.]; Andresen, Sabine [Hrsg.]; Moser, Vera [Hrsg.]; Prengel, Annedore [Hrsg.]:

Geschlechterforschung in der Kritik. Opladen ; Bloomfield Hills : Verlag Barbara Budrich 2005, S. 41-72 -

URN: urn:nbn:de:0111-opus-54164 - DOI: 10.25656/01:5416

https://nbn-resolving.org/urn:nbn:de:0111-opus-54164

https://doi.org/10.25656/01:5416

in Kooperation mit / in cooperation with:

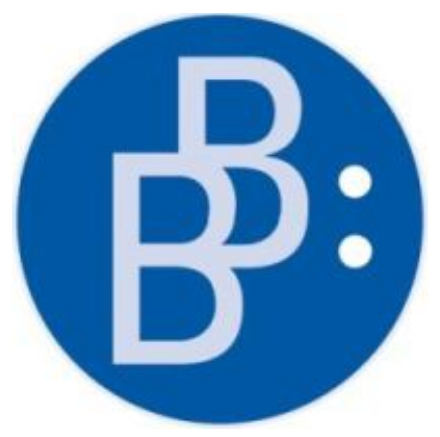

https://www.budrich.de

\section{Nutzungsbedingungen}

Gewährt wird ein nicht exklusives, nicht übertragbares, persönliches und beschränktes Recht auf Nutzung dieses Dokuments. Dieses Dokument ist ausschließlich für den persönlichen, nicht-kommerziellen Gebrauch bestimmt. Die Nutzung stellt keine Übertragung des Eigentumsrechts an diesem Dokument dar und gilt vorbehaltlich der folgenden Einschränkungen Auf sämtlichen Kopien dieses Dokuments müssen alle Urheberrechtshinweise und sonstigen Hinweise auf gesetzlichen Schutz beibehalten werden. Sie dürfen dieses Dokument nicht in irgendeiner Weise abändern, noch dürfen Sie dieses Dokument für öffentliche oder kommerzielle Zwecke vervielfältigen, öffentlich ausstellen, aufführen, vertreiben oder anderweitig nutzen.

Mit der Verwendung dieses Dokuments erkennen Sie die Nutzungsbedingungen an.

\section{Terms of use}

We grant a non-exclusive, non-transferable, individual and limited right to using this document.

This document is solely intended for your personal, non-commercial use. Use of this document does not include any transfer of property rights and it is conditional to the following limitations: All of the copies of this documents must retain all copyright information and other information regarding legal protection. You are not allowed to alter this document in any way, to copy it for public or commercial purposes, to exhibit the document in public, to perform, distribute or otherwise use the document in public.

By using this particular document, you accept the above-stated conditions of use.

\section{Kontakt / Contact:}

peDOCS

DIPF | Leibniz-Institut für Bildungsforschung und Bildungsinformation Informationszentrum (IZ) Bildung

E-Mail: pedocs@dipf.de

Internet: www.pedocs.de

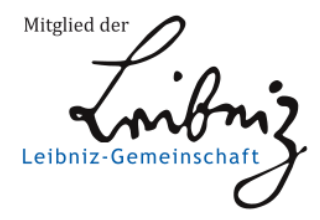


Geschlechterforschung in der Kritik 
Jahrbuch der

Frauen- und Geschlechterforschung in der Erziehungswissenschaft

herausgegeben von

Rita Casale

Barbara Rendtorff

Sabine Andresen

Vera Moser

Annedore Prengel

Folge 1/2005 
Rita Casale

Barbara Rendtorff

Sabine Andresen

Vera Moser

Annedore Prengel (Hrsg.)

\section{Geschlechterforschung in der Kritik}

Verlag Barbara Budrich

Opladen \& Bloomfield Hills 2005 
Gedruckt auf säurefreiem und alterungsbeständigem Papier.

Die Deutsche Bibliothek - CIP-Einheitsaufnahme

Ein Titeldatensatz für die Publikation ist bei Der Deutschen Bibliothek erhältlich.

Alle Rechte vorbehalten.

(C) 2005 Verlag Barbara Budrich, Opladen

www.budrich-verlag.de

\section{ISBN 3-938094-19-2}

Das Werk einschließlich aller seiner Teile ist urheberrechtlich geschützt. Jede Verwertung außerhalb der engen Grenzen des Urheberrechtsgesetzes ist ohne Zustimmung des Verlages unzulässig und strafbar. Das gilt insbesondere für Vervielfältigungen, Übersetzungen, Mikroverfilmungen und die Einspeicherung und Verarbeitung in elektronischen Systemen.

Umschlaggestaltung: disegno, Wuppertal - www.disenjo.de

Lektorat: Tina Jung

Satz: Verlag Barbara Budrich

Druck: DruckPartner Rübelmann, Hemsbach

Printed in Germany 


\section{Inhalt}

Grußwort der Vorsitzenden der Sektion Frauen- und

Geschlechterforschung in der DGfE

Editorial

\section{Beiträge}

Barbara Rendtorff

Strukturprobleme der Frauen- und Geschlechterforschung in der

Erziehungswissenschaft

Edgar Forster

Männerforschung, Gender Studies und Patriarchatskritik

Eva Breitenbach

Vom Subjekt zur Kategorie. Veränderte Denkfiguren

Fritjof Bönold

Zur Lage der pädagogischen Frauen- und Geschlechterforschung: bildungstheoretische Diskussionen

Susanne Maurer

Gedächtnisspeicher gesellschaftlicher Erfahrung? Zur politischen

Dimension von Frauen- und Geschlechterforschung

\section{Aus der Forschung}

Sabina Larcher

„Doing teacher" - professionelle Inszenierungen in Wechselwirkung von institutionellen Arrangements und sozialer Praxis

Marita Kampshoff

Geschlechterdifferenzen bei den Schulleistungen - empirische

Forschungen aus England und Deutschland im Vergleich 
Inga Pinhard

„Action is indeed the sole medium of expression for ethics “

Skizzen einer sozialen Ethik bei Jane Addams

\section{Rezensionen}

Christiane Micus-Loos

Rezension zu Eva Borst: Anerkennung der Anderen und das Problem des Unterschieds. Perspektiven einer kritischen Theorie der Bildung ...

Barbara Fontanellaz

Rezension zu Corinna Seith: Öffentliche Interventionen gegen

häusliche Gewalt. Zur Rolle von Polizei, Sozialdienst und

Frauenhäusern

Fritjof Bönold

Rezension zu Heide von Felden: Bildung und Geschlecht zwischen

Moderne und Postmoderne. Zur Verknüpfung von Bildungs-,

Biographie- und Genderforschung

Ankündigung der nächsten Bände

Band 2

Band 3

Über die AutorInnen 


\title{
Männerforschung, Gender Studies und Patriarchatskritik
}

\section{Edgar Forster}

\begin{abstract}
„Was bleibt dem abstrakten Denker, wenn er klug und überlegt Ratschläge gibt? Also immer über die Wunde von Bousquet, über den Alkoholismus von Fitzgerald und von Lowry, über den Wahnsinn von Nietzsche und von Artaud sprechen und dabei am Ufer bleiben? Zum

Fachmann für solches Geplauder werden? Nur wünschen, daß jene, die geschlagen wurden, nicht allzu tief sinken? Nachforschungen anstellen und

Sondernummern herausgeben? Oder versuchen, sich darin ein wenig selbst zu sehen, ein bißchen Alkoholiker, ein bißchen verrückt, ein bißchen selbstmörderisch, ein bißchen Guerillero sein, gerade genug, um den Riß auszudehnen, aber nicht zuviel, um ihn nicht unwiderruflich zu vertiefen? Wohin man sich auch wendet, alles scheint trostlos. In Wirklichkeit aber:

Wie sich an der Oberfläche halten, ohne am Ufer zu bleiben? Wie sich und dabei die Oberfläche und die ganze Oberflächenorganisation einschließlich der Sprache und des Lebens retten? Wie zu dieser Politik, zu dieser vollkommenen Guerilla gelangen?“
\end{abstract}

(Deleuze 1993, 197)

Das Verhältnis von Männerforschung, Gender Studies und Patriarchatskritik wird im vorliegenden Beitrag einer kritischen Analyse unterzogen, denn ungeachtet ihrer fortschreitenden Institutionalisierung gibt Männerforschung wenig Auskunft über sich selbst: über ihr Selbstverständnis, über ihr Verhältnis zum Feminismus und zum Patriarchat, über ihr Verhältnis zu Politik und Praxis. ${ }^{1}$ Anders als die Men's Studies in den USA, Australien oder in den

$1 \mathrm{Zu}$ den wenigen kritischen Standortbestimmungen zählt der Text Offensichtlich männlich (Tillner/Kaltenecker 1995). 
skandinavischen Ländern verdankt sich die Ausbreitung der deutschsprachigen Männerforschung in den 1990er Jahren vor allem den Gender Studies, die an vielen Universitäten umstandslos feministische Theorien und Praxen abgelöst und damit Unterschiede zwischen feministischen Theorien, Männerforschungen, Queer Studies eher nivelliert oder zugedeckt statt zum Gegenstand kritischer Analysen gemacht haben. Männerforscher profitieren in akademischen Feldern von Kämpfen, die die zweite Generation feministischer Theoretikerinnen seit mehr als dreißig Jahren führen. Sie nützen ihre Bühnen und werden in einer historischen Phase ermutigt und unterstützt, ,die' Männerforschung als Forschungsfeld weiter zu entwickeln, in der Frauenforschung und Frauenpolitik auf vielen Ebenen wieder in Verteidigungspositionen gezwungen werden und unter Legitimationsdruck geraten.

Dieser Kontext ist für eine kritische Selbstvergewisserung unabdingbar, denn mein Interesse gilt der Frage, wie eine Männerforschung aussehen müsste, die gemeinsam mit feministischen Theorien und Praxen eine umfassende Geschlechterdemokratie vorantreibt - ein Ziel, das zwar allgemein akzeptiert, aber ausbuchstabiert und in der Praxis eingefordert auch innerhalb der heterogenen Forschungslandschaft von Männerforschern und in den verschiedenen Feldern von Jungen- und Männerarbeit Gegenstand heftiger Debatten ist.

Unter der vagen Bezeichnung ,Theorie und Praxis von Männlichkeitskritik" bündle ich das zunehmende Interesse auch von Männern, Männlichkeit zum kritischen Gegenstand humanwissenschaftlicher Forschung zu machen. Dabei handelt es sich bis heute nicht um eine dem Feminismus vergleichbare ,Bewegung', die nachhaltige Veränderungen der Wissenschaftslandschaft bewirkt hat. Was man absehen kann, ist eine allmählich einsetzende Rezeption internationaler Männerforschung, die Sensibilisierung für Männlichkeit in einer Reihe von praktischen pädagogischen Feldern mit einem starken Fokus auf Jungen- und Männerarbeit. Ich beschränke mich hier auf einige theoretische Aspekte derjenigen Männerforschung, die im deutschsprachigen Raum rezipiert wird ${ }^{2}$ und vernachlässige aufgrund der Ungleichzeitigkeit der Diskurse und der unterschiedlichen thematischen und methodischen Zugänge Strömungen in anderen europäischen und außereuropäischen Ländern. ${ }^{3}$

2 Vgl. einführend BauSteineMänner (2001), Brandes (2002), Connell (1999), Döge (2001), Döge/Meuser (2001), Walter (2000).

3 Vgl. einführend Haywood/Mac an Ghaill (2003), Whitehead (2002). 


\section{Männlichkeitskritik: Fünf Thesen}

Ich möchte fünf Thesen diskutieren und damit eine Positionierung der Männerforschung vornehmen.

○ These 1: Männlichkeitskritik ist eine theoretische Praxis des Eingriffs.

○ These 2: Männlichkeitskritik unterhält ein kritisches Verhältnis zum Begriff Identität.

- These 3: Für Männlichkeitskritik bleibt das ,Patriarchat ${ }^{6}$ eine zentrale Analysekategorie.

○ These 4: Männlichkeitskritik ist weder Resouveränisierungs- noch Immunisierungsstrategie.

○ These 5: Männlichkeitskritik muss danach beurteilt werden, wie sie das Verhältnis zu feministischen Theorien und Praxen definiert.

\section{Positionierung}

Mein Nachdenken darüber, wie ich Männlichkeitskritik und damit meine eigene Forschungspraxis situieren möchte, hat eine ständige Referenz, ein wachsames und lustvolles Gegenüber: Donna Haraways Gedanken zum „situierten Wissen“. Aus diesem Text stammt auch der Satz: „Optik ist eine Politik der Positionierung“ (Haraway 1995, 86f.). Haraway argumentiert in ihrem Essay für die Verortung und Verkörperung von lokalisierbarem Wissen und verantwortbaren Erkenntnisansprüchen. Dieses Wissen ist utopisch und visionär. ${ }^{4}$ Aber Haraways Begriff der Vision bleibt noch mit der Körperlichkeit verschweißt und hat sich noch nicht in einen abstrakten, erobernden und unmarkierten Blick verwandelt, der Repräsentation erzeugt und ihr gleichzeitig entgeht. Da die Vision eine „Frage der Fähigkeit zu sehen“ und damit auch eine „Frage der unseren Visualisierungspraktiken impliziten Gewalt“ (ebd., 85) ist, bedeutet Vision immer eine bestimmte Art und Weise des Sehens. Die Vision hat ihren Wert in der partialen Perspektive, die sie ausdrücklich hervorhebt und anerkennt. Partialität heißt, man kann etwas auch anders sehen und muss sich für eine Sehweise entscheiden und für diese Entscheidung einstehen. So verbindet sich mit Haraways Begriff der Vision die Positionierung. 
Für Haraway ist Positionierung ${ }^{5}$ deswegen die entscheidende wissensbegründende Praktik, auf der Grundlage von Politik und Ethik. Eine visionäre Praktik entwickelt Sehpraktiken und Perspektiven, die nicht bereits im Voraus bekannt sind. Dabei bleibt sie aufmerksam für die Ränder des Sehens, für seine Ausschlussprozeduren, für das, was dem Sehen, das immer auch ein Fokussieren ist, entgeht.

Mit aller Vorsicht und das heißt gegen jede Absichtslosigkeit und Zufälligkeit führt Jacques Derrida das Wort position in seinen Text ein, das Dorothea Schmidt und Astrid Wintersberger mit „Setzung"6 übersetzen. Position hat bei Derrida eine präzise Funktion. Er benützt dieses Wort, um einen „Gestus aktiver Interpretation“ (Derrida 1986, 31) zu bezeichnen, der eine Reihe von Gesprächen über sein Werk charakterisiert. Die Positionierung gleicht einem ,Über-das-Ziel-Hinausschießen', denn eine Standortbestimmung des eigenen Werks kommt immer zu früh, sie ist immer unmöglich, da sie eine unabgeschlossene Arbeit rückblickend beenden und schließen würde. Deshalb hat es Derrida auch abgelehnt, in die deutsche Übersetzung der Gespräche, die vierzehn Jahre später publiziert werden, einzugreifen, denn jede nachträgliche Korrektur hätte eine absolute Autoritätsposition zum Text installiert: Das nachträgliche Zurechtrücken hebt den Autor in eine privilegierte Wahrheitsposition und suggeriert einen Ort der Wahrheit, von dem aus der Text ,richtig' gelesen werden kann. ${ }^{7}$

Bei Jacques Derrida (1986) kann man schließlich auch studieren, dass jeder Positionierung die Verausgabung eingeschrieben ist. Positionierung bedeutet, über das Ziel hinaus zu schießen; mehr zu sagen, als man weiß. Eine Positionierung öffnet das Feld, anstatt es abzuschließen, denn sie fordert heraus, eigene Positionen zu markieren, zu definieren - und damit sich auszusetzen und in Verhandlungen darüber einzutreten. Eine Position zu beziehen heißt nicht, der eigenen Forschung nachträglich Sinn einzuschreiben, sondern genau genommen: einen Akt zu setzen, einen Sprung zu wagen und einen Gedanken zu fixieren, der im Grunde durch seine Fixierung zu wirken beginnt, obgleich seine Fixierung ein Gewaltakt darstellt und zurückgenommen werden müsste, weil sie nicht haltbar ist. Aber sie löst nicht nur Kettenreaktionen aus, sondern sie zwingt sich zu verantworten und ist deshalb eine Praktik, die

Positionierung durchkreuzt das binäre Schema von Relativismus und Totalisierung, die sich spiegelbildlich zueinander verhalten (vgl. Haraway 1995, 84).

6 „Setzung“ - in der deutschen Ausgabe mit Anführungszeichen versehen, wohl um die Zurückhaltung und Schwierigkeit der Übersetzung angesichts des Bedeutungsüberschusses zu markieren.

7 „Dabei ist Derridas Lektüre Derridas in der gleichen Position wie unsere Lektüre Derridas." (Engelmann 1986, 17; Hervorhebung E.F.) 
der Ethik zuzurechnen ist. Sich zu positionieren ist eine Eingreifpraktik, eine theoretische Praktik, eine dekonstruktive Politik ${ }^{8}$

Düttmann (1992) weist auf die entgrenzende und verausgabende Kraft der Dekonstruktion hin. Notwendig sagt sie immer zu viel. Wenn es sich um Setzungen handelt, gibt es keine Begriffsapparate (vgl. Derrida 1986, 86). Sie funktionieren wie ein Supplement. Es (er-)öffnet das Feld, schießt über Grenzen hinaus, sprengt den Rahmen. Setzung ist „Setzung dieser Andersheit als solcher" (ebda., 177). In einem Brief an Houdebine (und dann im Gespräch mit Peter Engelmann) setzt sich Derrida (1986, 182f.) mit dem Wort position auseinander, und zwar mit „Setzung (der Andersheit)“: „1. Wenn die Andersheit des Anderen gesetzt (posée) ist, ja ausschließlich gesetzt ist, kommt sie dann nicht auf das Selbe zurück, zum Beispiel in der Form des ,konstituierten Objekts' oder des ,informierten Produkts', das mit einem Sinn versehen ist? Von diesem Gesichtspunkt aus würde ich sogar sagen, dass die Andersheit des Anderen in das Verhältnis das einschreibt, was in keinem Fall ,gesetzt ${ }^{\star}$ werden kann. Die Einschreibung, so wie ich sie in dieser Hinsicht definieren würde, ist nicht eine einfache Setzung: vielmehr das, wodurch jede Setzung durch sich selbst vereitelt wird (différance): Einschreibung, Markierung, Text und nicht nur These oder Themen-Einschreibung der These. [...] Und man kann den Begriff der Setzung (position) immer wieder umdefinieren, anhand desselben Wortes (Abzug, Verpflanzung, Ausweitung).“

Auf andere Weise zeigt Gilles Deleuze $(1997,101)$ in seiner Arbeit über David Hume die Bewegung einer verausgabenden Setzung im Verhältnis von Subjektivismus und Empirismus. „Es darf jedoch nicht übersehen werden, daß die Bewegung der Selbstentfaltung bzw. des Anders-Werdens eine doppelte ist: Das Subjekt geht über sich hinaus, das Subjekt reflektiert sich." Mit anderen Worten: „Glauben und Erfinden ist, was das Subjekt zum Subjekt macht" (ebda., 102). Schließlich besetzt die Einbildungskraft als Gegenstand und Methode der Kritik bei Dietmar Kamper $(1990,10)$ jenen Ort, von dem aus Antworten auf folgende Frage möglich werden: „Ist das, was einer mit seiner Erkenntnis anrichtet, ist überhaupt die Tatsache, daß einer so denkt, wie er denkt, mit Hilfe seiner eigenen Erkenntnis begreifbar?" Unübersehbar ist die Wiederaufnahme des Motivs der Nicht-Identität bei Adorno, die auf eine Theorie der Erfahrung zuläuft. Auch hier ergeben sich Parallelen zu Derrida $(1998,366,383)$ und zu Foucaults Der Mensch ist ein Erfahrungstier (1996). Erfahrung markiert in allen Fällen Kontingenz und Überschreitung.

8 „Warum soll man die Arbeit der Dekonstruktion aufnehmen, anstatt die Dinge so zu lassen, "wie sie sind? Usw. Nichts hier ohne ,Kraftakt', irgendwo. Die Dekonstruktion, darauf habe ich bestanden, ist nicht neutral. Sie interveniert" (Derrida 1986, 179f.). 
Dies ist die Bedingung für Veränderung, die noch nicht vollständig determiniert ist. Das aber bedeutet, dass wir in striktem Sinn genötigt sind, unmögliche Entscheidungen zu treffen.

\section{Eingriff}

Positionierungen und Setzungen anerkennen die partiale Perspektive und die damit verbundene Notwendigkeit, sich für eine Perspektive zu entscheiden und diese Entscheidung zu verhandeln. Positionierungen haben aber eine zweite Dimension: Jede Positionierung ist auch ein Eingriff in das Feld, das untersucht wird, denn die Vision, von der Haraway spricht, ist nicht nur die Fähigkeit zu sehen, sondern die Gewalt des Blicks zu sehen, indem der Blick auf sich zurückgeworfen wird, so dass er und seine Macht sichtbar werden. Weil der Blick und sein Feld nicht unabhängig voneinander existieren, weil also der Gegenstand einer Untersuchung mit seiner Repräsentation unauflösbar verknüpft ist, werden der Blick und das Subjekt des Blicks selbst zum Gegenstand der Untersuchung. Für Geschlechterforschung ist dies deswegen besonders bedeutsam, da der abstrakte, körperlose Blick ,die unmarkierte Position des Mannes und des Weißen" (Haraway 1995, 80) bezeichnet.

Deswegen lautet die erste These, dass Männlichkeitskritik eine theoretische Praxis des Eingriffs ist. Diese These enthält zum einen die Aussage, dass Theorie eine Aktivität darstellt, die die Beziehung zwischen Subjekt und Gegenstand der Untersuchung beschreibt, definiert und auch herstellt und damit das gesamte Feld produziert. Zum anderen bedeutet theoretische Praxis, ein Feld auf bestimmte Weise zu reartikulieren, also Verbindungen zwischen den Elementen eines Feldes herzustellen (und nicht nur abzubilden) (vgl. Hall 2000, 65). Würde sich Männlichkeitskritik als Männerforschung auf die ,blo-

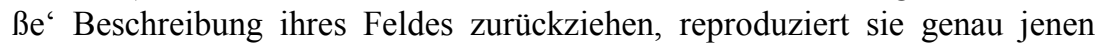
abstrakten phallozentrischen Blick, der selbst dem Gesehenwerden entgeht. In ein Feld einzugreifen bedeutet, die Macht des wissenschaftlichen Blicks sichtbar zu machen. In der Männerforschung paralysiert die Sprengkraft des Performativen, das die theoretische Seite des Eingriffs formuliert, das ,starke männliche Subjekt der Rede, das Selbstreflexion und Selbstkritik dazu nützt, Dominanz in verwandelter Form zu festigen. Männer erfahren dies für gewöhnlich so: Sie geben Positionen auf, sie stimmen der Kritik zu, dennoch bleiben ihre Positionen aus der Sicht feministischer Haltungen suspekt. Theoretisch geht es um die Frage, von welchem Ort aus männliche Subjekte spre- 
chen und - als kritische - sprechen können. Dabei geht es gerade nicht um eine politisch korrekte Position des Sprechens (wie manche Männer glauben machen wollen), sondern darum, die Position des Sprechens als eines männlich markierten Sprechens sichtbar zu machen - und damit die dem Eingriff eigene Form der Beschränkung und Verausgabung der theoretischen Aktivität.

\section{Who needs identity?}

„Wer braucht Identität?“ fragt Stuart Hall (1996) in einem Beitrag über das Verhältnis von Identität und Cultural Studies. Und wozu? Welche forschungsstrategische Bedeutung kommt der mit dem cultural turn verbundenen Wiederauferstehung des Begriffs Identität zu? Ähnlich äußert sich Lawrence Grossberg (1996a, 88): „Ich möchte zumindest die Frage stellen“, so Grossberg, „ob jeder Kampf auf dem Feld der Macht um Begriffe von Identitätsproblemen herum organisiert und verstanden werden kann und sollte und nahelegen, Identitätskonzepte neu zu formulieren." Grossberg interessiert sich dafür, wie Handlungsfähigkeit in spezifisch historisch-gesellschaftlichen Situationen hergestellt werden kann, wenn man nicht mehr davon ausgehen kann, dass es eine klare Trennlinie zwischen Mächtigen und Unterdrückten gibt und Widerstandspraktiken selbst in dem Machtfeld operieren, das sie bekämpfen. Dementsprechend stellt sich auch die Frage nach neuen Modellen politischer Gemeinschaften jenseits vorab festgelegter, stabiler Identitätskriterien.

Männlichkeitskritik unterhält, das ist meine zweite These, ein kritisches Verhältnis zum Begriff Identität. Das bedeutet, dass Männlichkeitskritik erstens danach fragt, was es bedeutet, Fragen von Männlichkeit und Männerpolitik als Identitätsfragen zu formulieren. Zweitens geht es um die Frage, wie Männerforschung das Verhältnis von Identität, Handlungsfähigkeit und Politik bestimmt. Ich werde die zweite These in drei Punkten artikulieren:

1. Zunächst werde ich die Funktion essentialistischer und nicht-essentialistischer Identitätskonzepte für die Männerforschung darstellen.

2. Dies mündet in den Vorschlag, die Begriffe „Interpellation“ (Althusser) und „Gabe“ (Derrida) für eine Geschlechtertheorie fruchtbar zu machen.

3. Im Anschluss an Grossberg werde ich schließlich diskutieren, wie eine Verschiebung der Grundlagen von Identität für Männlichkeitskritik genützt werden kann. 


\section{Essentialistische und nicht-essentialistische Identitätskonzeptionen}

Man kann, grob gesagt, zwei Paradigmen in der Identitätsdiskussion unterscheiden: ein essentialistisches und ein anti-essentialistisches Identitätsmodell. Zum ersten Modell zählen Formen personaler Identität, zum zweiten all jene Identitätsformen, die man auch als fragmentiert bezeichnen kann.

Personale Identität: Das erste Modell geht von einem unverbrüchlichen Identitätskern aus. Identität ist zeitüberdauernd und verbürgt Authentizität. Solche Identitätsmodelle haben in der deutschsprachigen Männerforschung und insbesondere in Theorien über Jungenarbeit ${ }^{9}$ eine ungeahnte Konjunktur. Unter dem Begriff personale Identität wird im Anschluss an die klassische Psychoanalyse und den Pragmatismus eine Tradition entwickelt, die (wie bei Erikson) Identität normativ entwirft und deswegen eng an Identitätskrise, Identitätsverlust und -diffusion anknüpft. Daraus speist ein Zweig in der Männerforschung und Männerarbeit immer wieder ihre Energie: Männliche Identität ist ständig bedroht, sie muss immer wieder neu erarbeitet und aufrechterhalten werden. Sie ist - per definitionem - krisenanfällig. Straub $(1998,95)$ nennt moderne Lebensbedingungen als unausweichliche Gefahr für Identität. „Als ein solches Konstrukt ist es etwas permanent Gefährdetes.“ Als Ursachen für männliche Identitätskrisen entdecken Männer regelmäßig den Feminismus, Frauen, die sich von ihren traditionellen Geschlechterpositionen emanzipieren, die Allmacht der Mütter, aus deren Abhängigkeit sich Jungen nicht lösen können, schließlich die angebliche Benachteiligung von Vätern in Scheidungsverfahren. Wenn sich Männlichkeit als relationaler Begriff über Weiblichkeit definiert, dann wird jede reale Veränderung von Geschlechterverhältnissen zu einer Gewinn-Verlust-Rechnung. Was Frauen gewinnen, verlieren Männer und die Emanzipation von Frauen ist dann unauflösbar mit männlichen Identitätskrisen verknüpft und nur noch als Bedrohungsszenario wahrnehmbar. Männliche Initiationsrituale sollen als besondere Facette männlicher Identitätsdebatten eine starke Männlichkeit unabhängig von unkalkulierbaren Wechselfällen der Geschlechterpolitik sichern. Bekannt geworden ist die mythopoetische Männerbewegung à la Robert Bly (1991). Jungen- und Männerarbeit sowie Männerforschungen, die daran anschließen, diagnostizieren Männer in der Krise und setzen auf starke Männlichkeit.

9 Eine Alternative dazu bietet Olav Stuve (2001), der Jungenarbeit mit Queer Theory verbindet (vgl. auch Bieringer/Buchacher/Forster 2000). 
Fragmentierte Identität: Das zweite Modell formuliert Identität nicht-essentialistisch. Es behauptet im Wesentlichen die Unmöglichkeit einer völlig konstituierten, von anderen klar unterschiedenen Identität. In den Cultural Studies sind diese Identitätsformen eng mit den Begriffen Fragmentierung, Hybridität und Diaspora verknüpft. Theorien über nicht-essentialistische Identitäten sind für die Diskussion des feministischen Subjekts in den 1980er Jahren, die mit Judith Butlers Gender Trouble ihren Höhepunkt und - vorläufigen - Abschluss erleben, zentral.

Für Männlichkeitskritik lassen sich im Anschluss an Butlers Ausarbeitung einer performativen Subjektkonzeption eine Reihe von Fragen stellen. Meine Bemerkungen und Fragen zu Butler sind im Gegensatz zu vielen Positionen in der Männerforschung ${ }^{10}$ nicht vom Wunsch getragen, poststrukturalistischen Positionen die Spitze zu brechen, sondern Männlichkeitskritik auf der Grundlage poststrukturalistischer und dekonstruktiver Theorieansätze weiterzuentwickeln.

○ Die deutschsprachige Männerforschung und Männerarbeit fällt zusammen mit der Transformation feministischer Theorien und der Durchsetzung von Gender Studies. Ich denke, dass Männlichkeitskritik diesen Umstand nicht außer Acht lassen darf und das Verhältnis von Männerforschung und Feminismus artikulieren müsste (vgl. unten).

- Butler hat zugespitzt formuliert, dass man nicht von vornherein eine Kategorie „Frau(en)“ annehmen kann und dass auch eine universale Struktur des Patriarchats problematisch sei. Wenn es die Kategorie „Frau(en)“" nicht gibt, so könne man auch nicht von den Männern (als Kategorie) sprechen, behaupten Georg Tillner und Siegfried Kaltenecker (1995). Sie halten die repräsentative Kategorie Männlichkeit für eine patriarchale Fiktion, die eine bindende (hegemoniale) Kraft für Männer entfaltet, die dem niemals erreichbaren Ideal von Männlichkeit nacheifern. Der Widerspruch zwischen dem Fan-

10 Robert W. Connell (1999) erwähnt sie im deutschen Standardwerk Der gemachte Mann. Konstruktion und Krise von Männlichkeiten überhaupt nicht und im neueren Buch The Men and the Boys nur am Rande, um ihr Performativitätskonzept zurückzuweisen (vgl. Connell 2000, 20). Holger Brandes (2002, 57ff.) diskutiert Butler kritisch. Problematisch sei, dass Butler „den Ort der Praxis falsch lokalisiert, indem sie soziale Praxis auf Diskurs und Text reduziert“”, „Soziale Praxis schließt“, so Brandes (ebd., 60f.), ,nämlich durch den Blick auf die Ebenen des Handelns in sozialen Kontexten den Körper und alle seine sinnlichen Qualitäten unmittelbar mit ein." Diese ,verkörperte“ soziale Praxis findet Brandes bei Connell und bei der Bourdieuschen Habitus-Theorie, die Brandes - der Titel seiner umfangreichen Arbeit, nämlich Der männliche Habitus, kündigt dies bereits an - die theoretische Grundlage seiner Männerforschung bietet. 
tasma einer vollen Männlichkeit und dem gelebten Mann-Sein erklärt sowohl männliche Gewalt, eröffnet aber auch ein Feld für andere Männlichkeitsstrategien. Die zweite Schlussfolgerung, die man aus der Transformation der Butlerschen These ziehen kann, ist die Zerstörung der Kategorie Mann selbst. Es gibt viele unterschiedliche männliche Lebensentwürfe, die nicht mehr durch eine gemeinsame Struktur verbunden sind. Damit verwandeln sich männliche Lebensentwürfe in bloße' Lebensentwürfe. Man mag zwar noch von hegemonialer Männlichkeit sprechen, aber sie ist allenfalls ein Bild, dem Männer nicht mehr notwendig unterworfen sind. Sie treten aus dem Männerbund aus und legen ihre Verantwortung für das Patriarchat ab. Eine latente Struktur, die die Gemeinsamkeit zwischen Männern als Männer herstellt, wird unterschlagen und damit geht der Blick für die fundamentale Ungleichheit zwischen den Geschlechtern zugunsten individueller Subjektpositionen verloren (vgl. Meuser/Behnke 1998, 12f.). Wenn man nun auch noch Butlers (vgl. 1991, 18) These, dass eine Frau zu ,sein', nicht alles ist, was man ist, in die Männerforschung übersetzt, wird Geschlecht als Strukturkategorie endgültig marginal. Ich werde diesen Faden mit der dritten These über das Patriarchat als Analysekategorie wieder aufnehmen. Hier ist zunächst zu beachten, dass Butler darauf insistiert, dass die Handlungsfähigkeit gerade nicht an ein herkömmliches, starkes feministisches Subjekt gebunden ist. Bedeutet nun die Dekonstruktion der Kategorie Mann den Abschied aus feministischer Politik? Folgt daraus schlicht die Negierung des Patriarchats? Und welche Bedeutung und Funktion hat dieses Patriarchat? Lässt es sich auch unter der Bedingung formulieren, dass es den Mann nicht gibt? Ist es möglich aus der Sicht von Männlichkeitskritik die Begriffe von Identität und Patriarchat zu reformulieren, die nicht hinter die Einsichten von Subjektkritik und Patriarchatskritik zurückfallen, ohne damit zugleich die Basis für Männlichkeitskritik aufzugeben? Was also bedeutet Butlers (1991, 35) Kritik, dass es falsch sei, ,von vornherein anzunehmen, dass es eine Kategorie ,Frau(en)" gibt“, für männlichkeitskritische Forschungen? Sind auch die Kategorien „Mann“ und „Männer“ unvollständige Kategorien, die ,als stets offener Schauplatz umkämpfter Bedeutungen“ (ebda.) dienen?

- Ein zentraler Aspekt der Butlerschen Dekonstruktion der sexgender-Dichotomie führt zu folgender Schlussfolgerung: „Die Begriffe Mann und männlich können dann ebenso einfach einen männlichen und einen weiblichen Körper bezeichnen wie umgekehrt die 
Kategorien Frau und weiblich" (Butler 1991, 23). Muss man sich demzufolge von der Differenz Mann/Frau verabschieden und stattdessen von weiblich und männlich markierten Subjektpositionen ausgehen? Donna Haraway $(1995,76)$ formuliert ihr Unbehagen unmissverständlich: „Diese Welt-als-Kode [...] ist ein hochtechnisiertes militärisches Feld, eine Art automatisiertes akademisches Schlachtfeld, in dem Leutpunkte, sogenannte Spieler, sich gegenseitig auflösen (was für eine Metapher!), um im Spiel um Wissen und Macht zu bleiben." Ist die Konstruktion von Subjektpositionen die einzige Möglichkeit, essentialistischen Fallen zu entgehen? Und was ist am Essentialismus die Falle? Um mit Haraway zu sprechen: Genügt es zu sagen, dass es theoretisch nicht ausgeschlossen werden kann, dass wir einen männlichen Körper mit dem verbinden, was wir Weiblichkeit nennen und umgekehrt, weil Weiblichkeit und Männlichkeit Konstruktionen sind? Würde das nicht dazu führen, dass kulturellen Konstruktionen letztlich eine Kraft zukommt, die einst die Biologie hatte? Als Naturalisierung wird die kulturelle Konstruktion als Schicksal erlebt, während es doch darum ginge, das Gesichtsfeld zu weiten, um anders zu sehen.

\section{,Gegebenes' ${ }^{6}$ Geschlecht}

Poststrukturalistische Identitätskonzeptionen wie diejenige von Judith Butler rufen vor allem auch in der Männerforschung immer wieder Irritationen hervor. Zum einen ist auf überraschende Weise das Subjekt immer noch sehr stark an das Individuum gekoppelt, auch wenn Subjektivität als Effekt diskursiver Strukturen gedacht wird. Nancy Fraser (vgl. 2001, 313) hat auch auf diesen Umstand aufmerksam gemacht und das Ausblenden von Intersubjektivität moniert. Die Intersubjektivität betrifft auch ein zweites Unbehagen mit poststrukturalistischen Identitätskonzeptionen, nämlich die Behauptung, dass nicht nur gender sondern auch sex konstruiert ist und dass die Konstruktionen theoretisch nicht an den Körper gebunden sind; vielmehr ist dieser selbst konstruiert. Barbara Duden (1993) hat Butler die Entkörperlichung der Frau vorgeworfen. Sie insistiert auf einer sinnlichen Dimension der Kategorie Geschlecht: „Stimme hat für mein Ohr immer Klang. Aus der Stimme klingt immer ein Mann oder eine Frau: beim ,ich'-Sagen braucht keine Stimme das Geschlecht anzugeben, denn es klingt aus dem gesprochenen ,ich'. Deshalb erschrecke ich, wenn hier ein stimmloser, stummer Diskurs, also reiner Text 
zur Grundlage des Wissens über Frauen gemacht wird“ (Duden 1993, 26). Nun ist der Klang der Stimme selbstverständlich auf die gleiche Weise diskursiviert, wie sex und gender. Dudens Argument kann Butlers Argumentation nicht aushebeln, es bestätigt vielmehr, dass man im Sprechen als Geschlechtswesen sichtbar und damit Subjekt wird. Worauf aber Duden aufmerksam machen will, ist, dass man doch immer schon als Geschlechtswesen ausgewiesen, markiert oder angerufen ist. Oder anders: Es erscheint, als sei Geschlecht immer schon gegeben, eine Gabe also, weshalb Kafka (1994) am 18. Januar 1922 in sein Tagebuch notieren kann: „Was hast Du mit dem Geschenk des Geschlechtes getan? Es ist misslungen, wird man schließlich sagen, das wird alles sein. Aber es hätte leicht gelingen können." Auch Butler (1991, 23) kommt auf das „gegebene“ Geschlecht zu sprechen: „Können wir noch von einem ,gegebenen' Geschlecht oder von einer ,gegebenen" Geschlechtsidentität sprechen, ohne wenigstens zu untersuchen, wie, d.h. durch welche Mittel, das Geschlecht und/oder die Geschlechtsidentität gegeben sind?" Während sich also Butler für die Konstruktionsweisen interessiert, die zum „gegebenen“ Geschlecht führen, möchte ich die Perspektive forcieren, was aus der Gabe des Geschlechts folgt. Dudens Beispiel ist nicht so sehr ein Beispiel für ihre eigene Argumentation, sondern ist vielmehr, wenn auch hinter ihrem Rücken, ein schönes Beispiel für ein intersubjektives Setting, durch das Subjektivität entsteht, und zwar auf doppelte Weise: Im Sprechen tritt man als Subjekt in Erscheinung. Zweitens aber ist die Anrufung ein wesentlicher Aspekt von Intersubjektivität. Für eine männlichkeitskritische Geschlechterpolitik erweist sich womöglich die Gabe oder die Anrufung als vielversprechender denn die Analyse der Praxen der Naturalisierung von Geschlecht. Ich möchte mich dabei in aller Kürze auf zwei Konzeptionen beziehen, auf die Interpellation von Althusser und die Gabe von Derrida.

Interpellation: Althussers $(1977,133)$ berühmter Satz über Ideologie lautet: „Die Ideologie repräsentiert das imaginäre Verhältnis der Individuen zu ihren realen Existenzbedingungen." Wenn Männlichkeit als Ideologie funktioniert, dann bezeichnet Männlichkeit ein bestimmtes Verhältnis von Individuen zu ihren realen Existenzbedingungen. Folglich definiert sich Männlichkeit nicht über ihre Relationalität zu Weiblichkeit und umgekehrt, denn diese wechselseitige Definition ist tautologisch und bringt keinen Erkenntnisgewinn. Männlichkeit und Weiblichkeit nehmen nicht durch gegenseitiges Verweisen Bedeutung an, sondern dadurch, dass sie unterschiedliche Verhältnisse zu Existenzbedingungen bezeichnen. Männlichkeit bezeichnet also eine Verknüpfungsregel. Althusser nennt dieses Verhältnis „Anrufung“/,,interpellation“. „Die Ideologie ruft die Individuen als Subjekte an“ (ebda., S. 140). Männ- 
lichkeit bezeichnet erstens den Akt, durch den ein Individuum zu einem Subjekt wird. Daraus geht zweitens nicht nur hervor, dass das ,ideologische Subjekt" eine Tautologie ist, sondern dass Männlichkeit eine Position und eine Operation bezeichnet. Und Männlichkeit bezeichnet drittens dasjenige Prinzip, das die Operation bewerkstelligt und Geschlechterpositionen produziert. Während die beiden ersten Teile der Definition von Männlichkeit auf Weiblichkeit übertragbar sind, schließt das dritte Merkmal von Männlichkeit, das Grosz Phallozentrismus nennt, Weiblichkeit kategorisch als relationale Kategorie aus, weil es nicht zwei strukturierende Prinzipien für die Herstellung eines bedeutungsvollen Rahmens geben kann. Die Herrschaft des heterosexuellen Systems mit Oppositionsbildungen, die durch Dominanz miteinander verbunden bzw. voneinander getrennt sind, verdankt sich einem strukturbildenden Zentrum. Der Effekt, der von diesem Prinzip für das Verhältnis der Geschlechter ausgeht, nämlich reale männliche Dominanz, rechtfertigt es, von Phallozentrismus, also von einem männlichen Prinzip zu sprechen. Praktiken, in denen Individuen als Subjekte auftauchen, sind auch jene Praktiken, in denen Individuen bestimmte Geschlechterpositionen einnehmen. Diese Positionierungen sind vorläufig, unabgeschlossen, aufgeschoben und sie sind eine „,ideologische Fantasie“: „(T)hey know that, in their activity, they are following an illusion, but still, they are doing it" (Žižek 1994, 33). Dass Gendering eine niemals abschließbare ideologische Fantasie darstellt, heißt nicht, dass sie keine materielle Existenz hat. Materiell ist eine Ideologie deswegen, weil sie nie für sich selbst vorkommt, sondern sich in ideologischen Staatsapparaten und ihren Praktiken realisiert. Deswegen sagt Althusser (1977, 137): „Eine Ideologie existiert immer in einem Apparat und dessen Praxis oder dessen Praxen. Diese Existenz ist materiell.“ Und zwar ,insofern seine Ideen seine materiellen Handlungen sind, die in materielle Praxen eingegliedert und durch materielle Rituale geregelt sind, die ihrerseits durch den materiellen ideologischen Apparat definiert werden, dem die Ideen dieses Subjekts entstammen“ (ebda., 139): Das heißt auch, dass Ideen keine ideale, geistige Existenz haben, sondern eine Existenz nur in konkreten Handlungen.

Die Gabe des Geschlechts: Poststrukturalistische Identitätskonzeptionen heften auf überraschende Weise Identität immer noch sehr stark an das Individuum, selbst dann, wenn Subjektivität als Effekt diskursiver Strukturen gedacht wird. Deswegen soll hier der Versuch unternommen werden, Gender an eine andere als individualistische Logik zu binden, und die These lautet, dass Geschlecht/Gender nach dem Prinzip von Ökonomie und Tauschlogik funktioniert. Ein Geschlecht im Sinne von Gender hat man nicht. Gender ist man auch nicht. Gender zirkuliert. Gender wird erworben, man bekommt es aufge- 
bürdet, es wird eingesetzt, getauscht und gegeneinander aufgerechnet. Nach dieser Logik kann Gender auch ein - willkommenes oder unwillkommenes Geschenk sein; ein Geschenk, dessen Annahme man zurückweisen oder das man annehmen oder zurückweisen kann oder vielleicht auch annehmen muss; eine Leihgabe, aber auch ein Auftrag. Geschlecht als Gabe erzeugt eine Verpflichtung, eine Verantwortung oder auch eine Schuld. Geschlecht als Gabe kann gewonnen und verspielt werden. Es gibt eine Vergeudung und Verschwendung von Geschlecht. Das Geschlecht als Gabe zu denken, heißt, das Geschlecht in einen Kreislauf der Ökonomie eingefügt zu sehen. „Die Gabe jedoch, wenn es sie gibt, bezöge sich ohne Zweifel auf die Ökonomie. Man kann nicht von der Gabe handeln, ohne von diesem Bezug auf die Ökonomie oder das Geld zu handeln, das versteht sich von selbst“(Derrida 1993, 16f.).

Aber die Gabe geht im Tausch nicht auf: „Aber ist die Gabe, wenn es sie gibt, nicht auch gerade das, was die Ökonomie unterbricht? Gerade das, was dem Tausch nicht mehr stattgibt, weil es den ökonomischen Kalkül suspendiert? Gerade das, was den Kreis öffnet, um sich der Reziprozität oder der Symmetrie, dem gemeinsamen Maß entgegenzustellen und so die Rückkehr in Rückkehrlosigkeit zu verkehren? Wenn es Gabe gibt, darf das Gegebene der Gabe (akkusativisch das, was man gibt, nominativisch das, was gegeben ist, die Gabe als das gegebene Ding oder als der Schenkungsakt) nicht zu dem Gebenden zurückkehren (sagen wir noch nicht zu dem Subjekt, zum Geber oder zur Geberin). Die Gabe darf nicht zirkulieren, sie darf nicht getauscht werden, auf gar keinen Fall darf sie sich, als Gabe, verschleißen lassen im Prozess des Tausches, in der kreisförmigen Zirkulationsbewegung einer Rückkehr zum Ausgangspunkt. Wenn die Figur des Kreises für die Ökonomie wesentlich ist, muss die Gabe anökonomisch bleiben. Nicht dass sie dem Kreis völlig fremd bliebe, aber sie muss dem Kreis gegenüber einen Bezug von Fremdheit bewahren, einen bezuglosen Bezug vertrauter Fremdheit. Und in diesem Sinne vielleicht ist die Gabe das Unmögliche“ (Derrida 1993, 17).

Die Gabe erlaubt es, das wäre eine provisorische These, mögliche Ausgänge für heterosexuelle Männlichkeiten aus dieser Tauschlogik zu denken und Geschlecht nicht mehr an Identitätslogiken zu binden, sondern dieses strikt ökonomisch zu denken. Geschlecht funktioniert dann wie das Geld bei Simmel. Es symbolisiert Wert, es macht sichtbar, man wird positioniert. Geschlecht wird ein Machtfaktor, der Kräfteverhältnisse bestimmt und Körper durchzieht. Die Formulierung von Geschlecht als Gabe im Anschluss an Derrida ist keine dritte oder vierte Lesart, eine völlig neue Position, die gegen alle anderen steht. Gerade dies wäre nicht die Position der Dekonstruktion. 


\section{Otherness, Produktivität und Räumlichkeit - Identität und Handlungsfähigkeit}

Grossbergs Kritik an Identitätskonzeptionen speist sich aus dem beschriebenen Unbehagen an einer individualistisch verkürzten Auffassung von Identität. Er weitet deshalb die kritische Analyse von Identität auf ihre modernen Grundlagen aus: auf Differenz, Individualität und Zeitlichkeit. Ihnen setzt er entgegen: Logiken der Otherness, der Produktivität und Rämlichkeit.

- Otherness: Die Logik des Anderen beruht auf der Annahme, dass der Andere unabhängig von einer spezifischen Beziehung zu dem Eigenen existiert und seinen eigenen Platz hat. Der Andere ist nicht mehr über eine Differenzrelation mit dem Eigenen verknüpft. Das bedeutet nun nicht, dass der Andere in essentialistischen Begriffen neu gefasst wird, sondern ,what it is can be defined by its particular (contextual) power to affect and be affected. That is, such view of otherness grant to each term an unspecified, but specifiable, positivity“ (Grossberg 1996a, 94). ${ }^{11}$ Affektive Logiken und Beziehungen bilden den Kern eines vom Eigenen unabhängigen Anderen. Bei Grossberg (vgl. 2000, 62) umfassen die Affekte Willenskraft, Stimmung, Beteiligung und Stärkung, die er vom Begehren und bloßen Vergnügen radikal trennt. Grossberg hat diese Unterscheidung in Bezug auf die Populärkultur getroffen und den Affekt als „Plateau der Effektivität“, ,die „Fähigkeit effektiv zu berühren und berührt zu werden“ (ebda.) bezeichnet. Somit wird Affekt zur Möglichkeit von Handlungsfähigkeit. Produktivität: Zur Logik der Identität gehört auch noch in der Version der radikalen Konstruktion - die Individualität. So formt der männliche Habitus die Männer und graviert sich ihrem Körper ein. Ob nun von einem authentischen Kern die Rede ist oder von der fragmentierten Identität, sie hat ihren Sitz im Individuum, das Identität, Bewusstsein und Handlungsfähigkeit artikuliert. Wenn nun Identität fragmentiert und unvollständig ist, stellt sich die Frage nach der Handlungsfähigkeit. Grossberg versucht nun, Handlungsfähigkeit von Identität zu lösen. Er fragt danach, was praktische Interventionen, die Realität transformieren, möglich

11 Grossberg verweist auf die rhizomatische Mannigfaltigkeit, die das Andere charakterisiert und den abstrakten Gegensatz zwischen Vielem und Einem vermeidet. Das Mannigfaltige, solchermaßen von jeder Dialektik gereinigt, organisiert keine Totalität mehr (vgl. Deleuze/Guattari 1997, 51). 
macht. Setzt Handlungsfähigkeit ein Subjekt voraus? Zumindest die strategische Supposition eines imaginierten ,vollständigen“ Subjekts? Diese Fragen werden durch das gemeinsame Modell der Repräsentation vereint, das die Handlungsfähigkeit als Repräsentation einer wie immer gefassten Identität versteht. Die Repräsentation ist umgekehrt mit einem Kausalmodell des Handelns verschweißt, das besagt, dass es für das Handeln Gründe gibt. Wenn man Handlungsfähigkeit allerdings nicht als Markierung des Subjekts begreift, sondern als konstituierendes Merkmal einer Zugehörigkeit, kurz als Produktion, dann löst sich Handlungsfähigkeit von Identität ab. Muss man als Mann entscheiden, ob man Feminist ist oder nicht? Ist es wichtig zu wissen (für andere oder mich selbst), ob die Unterstützung für Feministinnen an der Universität bloß strategisch ist oder aus - ja woraus eigentlich? - resultiert? Aus einer Überzeugung? Einer Ethik? Einem Wissen?

- Räumlichkeit: Der dritte Aspekt der Grossbergschen Dekonstruktion und Verschiebung der Identitätsproblematik betrifft die Logik der Zeitlichkeit, die er durch eine Logik des Räumlichen ersetzt. Hier geht es wesentlich um die Frage der Zugehörigkeit. Elspeth Probyn $(1995,53)$ zieht den Begriff belonging ${ }^{12}$ dem der Identität vor, weil es ihr so möglich wird, ,nicht in Standorten denken zu müssen, sondern mich an all den kleinen Linien der Sehnsucht zu orientieren“.

Auch Grossberg zielt nicht darauf ab, Identität aufzulösen und durch einen anderen Begriff zu ersetzen, sondern darauf, Handlungsfähigkeit und Identität von einander zu lösen und zu demonstrieren, dass sie nicht notwendig aufeinander rückführbar sind, ineinander übergehen und miteinander vernäht sind, sondern unterschiedlichen Logiken gehorchen und Bündnisse der Artikulation eingehen. „Social identities are themselves complex fields of multiple and even contradictory struggles; they are the product of the articulations of particular social positions into chains of equivalences, between experiences, interests, political struggles and cultural forms, and between different social positions“"(Grossberg 1996b, 156f.).

12 Probyn $(1995,54 \mathrm{f}$.) entfaltet das Bedeutungsspektrum von belonging: ,von ,jemandem oder einer Sache gehören' bis zu ,in die Gesellschaft passen“ oder ,Mitglied sein' geht es weiter zu belongings, was ,Besitztümer' und ,Gepäck' bedeuten kann. Für mich spiegelt belonging eine tiefsitzende Unsicherheit im Hinblick auf die Möglichkeit, wirklich dazuzugehören, wahrhaft in eine Umgebung zu passen, wider. Der Ausdruck belongings andererseits verweist auch auf die vielfältigen Arten, die es von dieser Sehnsucht, in eine Umgebung zu passen, gibt: freudvolle, schmerzliche, zum Scheitern verurteilte. Auf direktere Weise evoziert belonging Bilder des Weggehens an sich - es bezeichnet die Zwischenräume zwischen Sein und Sehnen, Kommen und Gehen.“ 


\section{Geschlechtsidentität und Männlichkeitskritik - Resümee}

Männlichkeitskritik positioniert sich zu Identität folgendermaßen: Nicht Identität, sondern Handlungsfähigkeit, Positionierung und Eingriff sind zentrale Begriffe für die Männerforschung. Wenn Handlungsfähigkeit mit Positionierung und Eingriff einhergeht, wenn Positionierung und Eingriff immer ein unbestimmtes $\mathrm{Ma} \beta$ an Verausgabung bedeuten, und zwar in der Form des Einsatzes von etwas, das nicht kontrollierbar ist, dann bedeutet es, dass Handlungsfähigkeit nicht in Relation zu Identität steht, sondern zu Ethik und Politik. Selbstverständlich hat Handlungsfähigkeit eine normative Grundlage, die sich aber in der Positionierung immer selbst überschreitet. In dieser Nichtkontrollierbarkeit der Überschreitung taucht auf, was Grossberg Otherness nennt.

Der Unterschied zu Judith Butler ist, auch wenn beide Konzeptionen antiessentialistisch konzipiert sind, eklatant. Das lässt sich am Beispiel des Wertes von Strategien sehen. Butler $(1991,20)$ betont, dass sich die Probleme der Repräsentation auch dann nicht verringern, ,wenn nur zu ,strategischen“ Zwecken an die Kategorie ,Frau(en) ${ }^{`}$ appelliert wird. Denn Strategien haben stets Bedeutungen, die über die angestrebten Ziele hinausgehen." Dieses Problem stellt sich nur dann, wenn man erstens die Frage der Handlungsfähigkeit an Identität und damit an Repräsentation bindet und zweitens, wenn man damit gleichzeitig unterstellt, dass es eine ,wahre', ,richtige' oder ,authentische ' Repräsentation für Praxen geben muss. Bei Butler hat ,strategisches Handeln" implizit etwas vom Vorwurf der Täuschungsabsicht, dem Spiel mit gezinkten Karten. Hier taucht noch einmal eine Form des „reinen“ Denkens auf. Das „reine“ Denken will sich als „starkes“ Denken auszeichnen, als ein Denken, das die unumstößliche Grundlage für Handlungen bildet. Unumstößlich heißt, es soll für das Handeln die zwingende Grundlage schaffen. Zwingend bezieht sich auf den Anderen, der dadurch das Handeln als ein zwingendes anerkennen soll. Strategie wird so zu einer Untugend der Täuschung, auch der Selbsttäuschung, der Schwäche des Geistes, zur Manipulation des Anderen, zur Vortäuschung falscher Absichten. Das starke Denken hingegen appelliert an eine bestimmte Logik von Objektivität, die an eine starke Subjektposition gebunden ist, der Haraway $(1995,86)$ 'ihren Objektivitätsbegriff entgegenstellt: „Das Versprechen der Objektivität liegt darin, dass wissenschaftlich Erkennende nicht die Subjektposition der Identität suchen, sondern die der Objektivität, das heißt der partialen Verbindung. Es gibt keine Möglichkeit, an allen Positionen zugleich oder zur Gänze an einer einzigen, privilegierten (unterdrückten) Position zu ,sein', die durch Geschlecht, ethnische und nationale Zugehörigkeit und Klasse strukturiert wird.“ 
Haraways „Vision“ findet ihren Abdruck auch in Drucilla Cornells (1991, 131) „ethical feminism“, ,[that] 'envisions' not only a world in which the viewpoint of the feminine is appreciated; ethical feminism also 'sees' a world 'peopled' by individuals, 'sexed' differently, a world beyond castration. Through our 'visions' we affirm the 'should be' of a different way of being human. The 'goal' of ethical feminism, which 'sees' the 'should be' inherent in the feminine viewpoint, is not just power for women, but the redefintion of all of our fundamental concepts, including power.“

Singularität ist für Grossberg (vgl. 1996a, 103) im Anschluss an Deleuze und Guattari und an Agamben ein Begriff, der Zugehörigkeit ohne Identität bezeichnet. Singularität ist eine Existenzweise, die weder universal noch partikular gedacht werden kann. Das Beispiel als Beispiel wäre für Agamben eine solche Existenzweise. Das Beispiel ist ein Zwischending, nicht bloß zufällig, aber auch nicht das Wesentliche, der Kern. Beispielhafte Praxis wäre eine werdende Praxis, die sich aber niemals ganz erfüllt.

\section{Männlichkeitskritik ist Patriarchatsanalyse}

Sexismus ist Sexismus erst durch eine zweite Form der Unterdrückung. Grosz nennt das dem Sexismus zugrunde liegende System ,patriarchal“. „Oberhalb und jenseits partikularer, konkreter sexistischer Handlungen liegt eine Struktur, die systematisch Männlichkeit in positiven und Weiblichkeit in negativen Termini wertet. Patriarchalismus ist damit ein struktureller Modus der Sozialorganisation, der Männer und Frauen in gesellschaftlichen, ökonomischen und interpersonellen Verhältnissen unterschiedlich positioniert. Er besteht nicht aus empirischen Handlungen; es ist eine latente Struktur, die diese individuellen Akte ermöglicht und sie in einer systematischen Form organisiert und den praktischen Kontext sowie die Bedeutungszusammenhänge sexistischer Ungleichheit liefert. Selbst wenn alle empirischen Beispiele für Sexismus wegfallen würden, würde die positionelle Unterdrückung von Frauen unverändert bleiben. Sie besteht nicht nur aus der unterschiedlichen und ungleichen Behandlung der beiden Geschlechter, sondern auch aus den differenten Bedeutungen und Wertungen, die ihnen zugeschrieben werden, auch wenn sie sich auf gleiche Weise verhalten. Was bei Männern als Zeichen von Stärke gewertet wird, würde bei Frauen als Aggressivität betrachtet werden, selbst bei identischem Verhalten. [...] Patriarchale Strukturen sind nicht unwandelbar, sondern historisch variabel und funktionieren in soziographisch spezifischen Kulturen auch auf spezifische Weise; dennoch behalten sie stets die 
Bestimmung, männliche Suprematie aufrechtzuerhalten und fortzuführen“ (Grosz, zit. nach Knapp 1993, 311f.). Als soziale Strukturkategorie ${ }^{13}$ existiert Geschlecht nur, wenn gesellschaftliche Macht entlang von Personengruppen mit klar definierten Gruppeneigenschaften (in diesem Fall Geschlecht) organisiert ist. Die Frage ist also nicht, ob es das Patriarchat gibt oder nicht, sondern wie das Patriarchat gedacht werden muss, um einerseits Sexismus zu ermöglichen, andererseits die vielfältigen Machtverhältnisse zwischen Männern und Frauen und innerhalb von Männern und Frauen zu repräsentieren. ${ }^{14}$

Es geht also um eine Theorie, die die Beweglichkeit des Patriarchats ausdrückt. Mann und Männlichkeit sind unzulängliche Verkürzungen, die den trügerischen Schein einer kollektiven Identität erzeugen. Aber löst die Vervielfältigung der Positionen dieses Dilemma? Repräsentieren viele Männlichkeiten eine Kategorie, die plausibel macht, dass Frauen nach wie vor in vielen Lebensbereichen diskriminiert werden? Muss die Analyse von Männlichkeit nicht bei dem Widerspruch einsetzen, dass den vielen widerstreitenden Männerinteressen ein institutionalisierter hegemonialer patriarchaler Apparat in die Hände arbeitet? ${ }^{15}$ Sind die vielfältigen männlichen Identitätsverhandlungen in einen phallozentrischen Rahmen eingebettet, der in dem Maße wirkt, wie er unsichtbar bleibt? Ist das Allgemeine männlich, unabhängig davon, wie widersprüchlich Männer untereinander handeln? So sehr auf der einen Seite herkömmliche theoretische Unterscheidungen und Kategorien dekonstruiert werden, so kommt man andererseits nicht umhin zu sehen, dass der Alltag immer noch von traditionellen Geschlechterrollenverteilungen geprägt wird.

13 Geschlecht als (soziale) Strukturkategorie ist Becker-Schmidt (vgl. 1993) zufolge eine von sieben Komponenten des Terminus' Geschlecht.

14 Genau so wie Butler das Subjekt des Feminismus, die Kategorie ,Frau(en)' kritisiert, kritisiert sie auch die Struktur des Patriarchats: „Die politische Annahme, dass der Feminismus eine universale Grundlage haben müsse, die in einer quer durch die Kulturen existierenden Identität zu finden sei geht häufig mit der Vorstellung einher, dass die Unterdrückung der Frauen eine einzigartige Form besitzt, die in der universalen oder hegemonialen Struktur des Patriarchats bzw. der männlichen Herrschaft auszumachen sei. Allerdings ist die Vorstellung von einem universalen Patriarchat in den letzten Jahren auf breite Kritik gestoßen, weil sie unfähig ist, den spezifischen Vorgehensweisen der Geschlechterunterdrückung in den konkreten kulturellen Zusammenhängen Rechnung zu tragen“ (Butler 1991, 18f.).

15 Einen Beitrag, auf den ich hier nicht näher eingehen kann, liefert Iris Marion Young, die im Anschluss an Sartres Unterscheidung zwischen Gruppe und Serie die Problematik dessen, was eine Gruppe definiert, aufzulösen versucht und damit ein zentrales Thema feministischer Politik angeht: „Einerseits verliert eine feministische Politik ihre Besonderheit, wenn sie nicht in irgendeiner Weise davon ausgeht, daß es ein gesellschaftliches Kollektiv 'Frauen' gibt. Andererseits untergräbt aber anscheinend jeder Versuch, die Eigenschaften dieses Kollektivs zu definieren, die feministische Politik, weil dadurch notwendig manche Frauen ausgeschlossen werden, die der Feminismus einbeziehen müsste“ (Young 1994, 24). 


\section{Patriarchatsdividende}

Robert Connell (1999) nennt die männliche Teilhabe am Patriarchat „Patriarchatsdividende“" um auszudrücken, dass wir Männer, ob wir wollen oder nicht, in privilegierte Positionen gesetzt sind. Es gibt weder den unschuldigen, das heißt geschlechtsneutralen Blick, noch ein solches Handeln. Was immer ein Mann sagt und tut, in einem patriarchalen Kontext wird es als geschlechtliches Handeln bedeutsam: Es kann als typisch männliches Handeln wahrgenommen werden oder auch als irritierendes Handeln, da es den unterstellten Normen nicht entspricht, etc. Aber es wäre eine Illusion zu glauben, dass es ein Sprechen vor der sexuellen Markierung gibt. Unklar bleibt, auf welche Weise unterschiedliche Formen der Teilhabe zustande kommen. Und es hat den Anschein, als habe die Veränderung von Männlichkeitsbildern und männlichen Handelns in den vergangenen Jahrzehnten wenig an der Wirkkraft des Patriarchats verändert, so als funktioniere dieses wie ein Selbstläufer unterhalb der sich rasch ändernden Geschlechterpraktiken an der Oberfläche der Gesellschaft. ${ }^{16}$

\section{Die Beweglichkeit des Patriarchats}

Um die Funktionsweise des Patriarchats zu verstehen, ist es wichtig, seine vereinheitlichende Funktion und seine verkettende und substituierende Beweglichkeit zu verstehen. ${ }^{17}$ Darin gleicht das Patriarchat der Funktionsweise des Rassismus. Die vereinheitlichende Funktion des Rassismus besteht in seiner Ausgrenzung und sozialen Normalisierung, seine Beweglichkeit aber darin, dass er seine Funktion auf allen gesellschaftlichen Feldern durchsetzt und so an Stärke und Legitimation gewinnt. Soziale Identitäten sind jene komplexen Schauplätze, die die Struktur für die Beweglichkeit des Rassismus bilden, denn auf ihnen finden vielfache und widersprüchliche Kämpfe statt, bei denen

16 Ein zweites Modell, um die Frage männlicher Teilhabe am Patriarchat zu lösen, ist das Habitus-Konzept von Bourdieu, auf das sich so unterschiedliche Theoretiker wie Ralf Bohnsack (2001), Lothar Böhnisch (2001), Holger Brandes (2002) und Michael Meuser (1998) beziehen. Parallelen zu Connells Patriarchatskonzept sind unübersehbar, auch wenn Connell Bourdieus Habitus-Konzept als zu funktionalistisch zurückweist (vgl. Brandes 2002, 61, Anm. 3). Ich kann hier nicht näher auf das Habitus-Konzept und seine Probleme eingehen (vgl. Forster/Rieger-Ladich 2004).

17 Auf ähnliche Weise habe ich versucht, das Problem des Rassismus und der Rassismusprävention anzugehen (vgl. Forster 2001). 
unterschiedliche Differenzen miteinander verhandelt werden. In diesen Identitätskämpfen verknüpft sich der Rassismus mit dem Nationalismus, mit dem Plädoyer für kulturelle Differenz, mit Argumenten zur Sprachgemeinschaft, mit Wirtschaftslogik, gleichermaßen mit Sexismus und Antisexismus, mit Generationenkonflikten usw.

Die Beweglichkeit des Patriarchats besteht in seiner mühelosen Artikulationsfähigkeit. Die Theorie der Artikulation beschreibt die kontingente (nichtkausale) Verknüpfung machtvoller kontextgebundener und kontextproduzierender ethnischer, Generationen-, Geschlechter- und Klassen-Differenzen (vgl. Hall 2000). Artikulation heißt Verbindung und Substitution einzelner Elemente einer Kette. Laclau (1997) nennt sie Äquivalenzkette. Auf diese Weise werden in alltäglichen Praktiken soziale Identitäten (re-)produziert und (re-)präsentiert. Die Konstruktion des ,Wir' und der Anderen wird so zu einer beständigen Re-Artikulation machtvoller Inklusions- und Exklusionsprozeduren.

Prävention gegen Exklusion und soziale Normierung operieren gegen die Verkettungslogik und seine Mythenproduktion. Das Geschlechterverhältnis ist ein Feld, auf dem Exklusion und soziale Normierung besonders reibungslos funktionieren, weil hegemoniale Männlichkeit und die sie stützenden Strukturen vielfach unsichtbar bleiben.

\section{Männlichkeit und Äquivalenzlogik}

Wenn man Männlichkeit als Ideologie begreift, dann gelingt es, so meine These, ein nicht-essentialistisches Männlichkeitskonzept mit struktureller männlicher Dominanz zusammen zu denken. Wenn man Männlichkeit als Ideologie versteht, weist man die Behauptung eines außerdiskursiven Standpunktes zurück. Zwar wird der Anschein erweckt, dass es eine volle Bedeutung von Männlichkeit gibt, tatsächlich aber wird diese Bedeutung durch eine „fiktive Fixierung" hergestellt, die Männlichkeit notwendig als falsche Repräsentation installiert. Die Illusion von der Wahrheit des Geschlechts verschleiert die Tatsache, dass die fiktive Fixierung über Inklusions- und Exklusionsmechanismen gewaltsam hergestellt wird. Männlichkeit als Ideologie zu begreifen, ist der Versuch, Männlichkeit als Funktionsweise bzw. als Operation zu fassen. Zusammengefasst lässt sich Männlichkeit folgendermaßen beschreiben: 
○ Männlichkeit wird durch Praktiken produziert, verändert und repräsentiert.

- Mit bedeutsamen Praktiken situiert sich ein Individuum als Subjekt in einem Diskurs.

- Die historisch konstituierten Bedeutungen von Praktiken sind geschlechtlich, ethnisch etc. kodiert, sodass man in diesem Diskurs immer nur und zugleich als Geschlechts-, Klassen-Subjekt etc. auftaucht.

○ Männlichkeit wird durch eine Kette von Bedeutungen - Laclau nennt sie Äquivalenzkette - repräsentiert. Keine Bedeutung ist imstande, Männlichkeit als Ganzes zu fassen.

- Ideologisch ist jede Bedeutungsfixierung, denn jede volle Bedeutung ist disloziert. In der falschen Repräsentation besteht die der Ideologie immanenten Idee der Verzerrung.

Indem Männlichkeit Bedeutungen fixiert, produziert sie Differenzen, die wiederum Macht organisieren. Männlichkeit unterscheidet sich von anderen Geschlechterpositionen durch die Art und Weise der Differenzproduktion. Männlichkeit funktioniert als Ideologie, weil Männlichkeit etwas anderes bedeutet als sie vorgibt zu sein: Essenz, Substanz, Eigenschaft. Mit Männlichkeit verbindet sich die Vorstellung eines Erkenntniszuwachses über Identität, während sie tatsächlich das Scheitern dieser Erkenntnis repräsentiert (vgl. Copjec 1995).

Ideologie lässt sich auf drei Achsen situieren: Erstens ist Ideologie ein Gefüge von Ideen: Theorien, Überzeugungen etc. Zweitens ist sie die Materialität dieser Ideen: ideologische Staatsapparate (religiöse Institutionen, Familie etc.). Drittens ist Ideologie „, ,spontane“ Ideologie, die im Herzen der gesellschaftlichen ,Realität' selbst am Werk ist“" (Žižek, zit. nach Laclau 1997, 45). „Spontane Ideologie“ realisiert sich auf der Ebene alltäglicher Praktiken und gesellschaftlicher Institutionen. Als „legitimierte Situationsdefinitionen“ (Falk/Steinert 1973, 39) produzieren Institutionen Regeln für Zugehörigkeit. Sie funktionieren immer über Inklusion und Exklusion (vgl. Douglas 1991).

Auf diese Weise ergibt sich eine Definition von Männlichkeit durch das Zusammenspiel von drei Dimensionen: Männlichkeit definiert sich durch alltägliche Praktiken, deren Bedeutungen durch diskursive Rahmenbedingungen hergestellt und verändert werden und sich in institutionellen Materialisierungen niederschlagen. Diese Dreiteilung findet sich in Grosz' Analyse von Machtverhältnissen wieder: Sexismus, Patriarchalismus und Phallozentrismus. Sexismus drückt Männlichkeit in Form konkreter, alltäglicher Praktiken aus. Patriarchalismus unterstützt sexistische Praktiken durch Institutionen. 
Phallozentrismus bezeichnet die legitimierte Autorität, die Regeln vorgibt, nach denen Institutionen und Praktiken funktionieren und ihre Bedeutung im (Geschlechter-)Diskurs annehmen. Daraus folgt, dass Männlichkeit nicht nur ein Element in der Geschlechterdifferenz ist, sondern das Strukturierungsprinzip, das die Differenz bestimmt.

Das Argument, Männlichkeit funktioniere als Ideologie, enthält zwei Annahmen: Erstens macht das Reden von Ideologie nur Sinn, wenn es einen Bereich des Nichtideologischen gibt. Zweitens: Der Begriff Ideologie enthält die Idee der Verzerrung in Gestalt der Unwahrheit oder Täuschung. Die sex-gender-Trennung hat es ermöglicht, sex als außerdiskursiven Fixationspunkt aufrechtzuerhalten. Dass eben darin die Ideologie der Geschlechterkonzeption bestand, hat Judith Butler $(1991,26)$ nachgewiesen. Es geht in der Folge darum zu sehen, dass die Dominanz des Männlichen durch den Anschein erzeugt wird, es handle sich um Naturwüchsigkeiten, die deswegen als solche nicht wahrgenommen werden. Diese ideologische Täuschung unterscheidet Männlichkeit grundlegend von Weiblichkeit.

Die Annahme, dass die Idee eines außerdiskursiven Standpunktes „die ideologische Täuschung schlechthin“ (Laclau 1997, 48) sei, führt Laclau zu folgender Frage: Wie kann man an einem Begriff der Verzerrung und damit am Begriff der Ideologie festhalten, ohne den für den Begriff der Verzerrung notwendig außerdiskursiven Standpunkt einnehmen zu müssen? Nicht nur das Außerdiskursive, sondern auch das Diskursive ist Ideologie, denn das Soziale muss als symbolisches System vorgestellt werden, als Netz von Signifikanten, unter die sich frei flottierende Bedeutungen schieben. Will man Bedeutungen fixieren, muss dieses System zum Stillstand gebracht werden, indem man einem Signifikanten genau ein Signifikat zuordnet. Diese Fixierung nennt Laclau Schließung. Sie ist notwendig ideologisch.

Die Verzerrungsoperation besteht darin glauben zu machen, es gäbe eine ursprüngliche, unverzerrte Bedeutung. Die Verzerrung besteht darin, dass auf etwas „fundamental Gespaltenes die Illusion einer Fülle und Selbsttransparenz" (ebd., 50) projiziert wird. Männlichkeit produziert die Illusion einer vollen Bedeutung von Männlichkeit. Zur Verzerrung gehören die Dislokation der vollen Bedeutung und die Verschleierung der Dislokation. Damit es Bedeutung gibt, braucht es eine „fiktive Fixierung“ (ebda., 51). Sie ist „falsche“ Repräsentation, das, „woraus jede Identität ihre fiktive Kohärenz zieht“. Männlichkeit gibt auf diese Weise der geschlechtlichen Identität eine fiktive Kohärenz. Da die Schließung notwendig aber unmöglich ist, hat sie keinen eigenen Inhalt. Sie „zeigt sich lediglich durch ihre Projektion auf ein von ihr verschiedenes Objekt" (ebda., 52). Zwischen diesem Objekt und der SchlieBungsoperation gibt es eine wechselseitige Abhängigkeit. Ideologisch werden 
Praktiken, wenn diese etwas über sie Hinausgehendes und von ihnen selbst Verschiedenes zu inkarnieren beginnen. Damit Ideen mehr als diese sind und Ideologien werden, muss es eine „Horizont-Dimension“ geben, innerhalb derer einzelne Praktiken als Elemente fungieren und Bedeutung durch ihre Opposition gegenüber allen anderen Elementen annehmen. Eine Vielzahl unterschiedlicher Praktiken können ein äquivalenter Name für Männlichkeit abgeben: ... $\leftrightarrow$ physische Stärke $\leftrightarrow$ Herrschaft $\leftrightarrow$ Dominanz $\leftrightarrow$ Gewalt $\leftrightarrow$ Durchsetzungsvermögen $\leftrightarrow$ Erfolg $\leftrightarrow$ Karriere $\leftrightarrow$ Geld $\leftrightarrow$ Heterosexualität $\leftrightarrow$... Ist Männlichkeit nun ein flottierender Signifikant, der überdeterminiert ist und Sinnüberschuss produziert, oder ist er ein leerer Signifikant, ein Signifikant ohne Signifikat? Laclau $(1997,55)$ zufolge ist der „flottierende Charakter eines Signifikanten die einzige Erscheinungsform seiner Leere“. Männlichkeit ist ein flottierender Signifikant und deshalb imstande, eine endlose Äquivalenzkette zu erzeugen. Dabei werden ständig Oppositionen gebildet, so dass die Behauptung gerechtfertigt erscheint, dass Bedeutungsproduktionen auch über oppositionserzeugende Inklusions- und Exklusionsmechanismen funktionieren. Äquivalenzketten haben eine doppelte Ausdehnung: Elemente verknüpfen sich zu einer Kette. Gleichzeitig produzieren aber Elemente oppositionelle Elemente, die sich selbst zu einer Art „oppositioneller Kette" verknüpfen. Auf diese Weise werden über Äquivalenzketten und das heißt durch Identitätspraktiken permanent Grenzen produziert und verändert.

Hegemonie setzt die Logik des leeren Signifikanten voraus. Männlichkeit tendiert zur Hegemonie genau dann, wenn sie nicht nur männliche Identiät fixiert, sondern überhaupt Identität; wenn sie also auch Weiblichkeit und andere Männlichkeiten fixiert; wenn sie schließlich das Geschlechterverhältnis regelt; wenn sie, kurz gesagt, den Diskurs der Geschlechter fixiert und wenn sie darüber hinaus, jenseits des Geschlechterdiskurses, Normalität regelt., 18 Dabei muss aber berücksichtigt werden, dass der Hegemonie produzierende Prozess auch sein Gegenteil ermöglicht: Zerstreuung.

18 „Hegemonie ist eine Angelegenheit von Zustimmung, nicht von Konsens, und es ist eine besondere, historisch neue Form des Kampfes um Macht, weil sei vom Aufkommen ,der Massen“ als politisches und kulturelles Subjekt abhängt“ (Grossberg 2000, 58f.). 


\section{Männlichkeitskritik und ihr Verhältnis zu feministischen Theorien und Praxen}

Wenn man Modelle zur Jungen- und Männerarbeit sowie zur Männerforschung einer kritischen Reflexion unterzieht, dann stellt man rasch eine Bruchlinie fest, die besonders sensibel zu sein scheint und deshalb für die Analyse von Männlichkeitskritik zielführend und wertvoll ist. Diese Bruchlinie betrifft das Verhältnis von Männerarbeit und Männerforschung zur feministischen Theorie und Praxis, also zu jenem vielgestaltigen Diskurs, den (zumindest einige) Männer ab sofort mitbestimmen und mittragen, aber auch verändern und umdefinieren wollen. Leitbegriffe dieser Bruchlinie sind: Feminismus, Antisexismus und Patriarchatskritik.

Männlichkeitskritik heißt zuerst, ihr Verhältnis zum Feminismus zu befragen. Die anderen Bruchlinien und Problemfelder stehen damit in engem Zusammenhang (vgl. Forster 2002).

In der Einführung zum Buch Nur Macher und Macho? Geschlechtsreflektierende Jungen- und Männerarbeit von Kurt Möller $(1997,11)$ ist zu lesen: „Jungen- und Männerarbeit hegt nicht den Anspruch, Feministen heranzuziehen, ein Unterfangen, das ohnehin paradox wäre." Es ist erstaunlich festzustellen, dass eine Reihe von Ansätzen in der Jungen- und Männerarbeit mit einer Distanzierung zu feministischer Theorie und Praxis und mit einem Zurückweisen von Forderungen von Frauen beginnen. Der Begriff „Feminist“ erfährt - hier - eine ambivalente Wertung. Zumindest in Form der losen Assoziation taucht eine typisch homophobe Strategie auf: Jungen und Männer werden abgewertet, indem sie ,verweiblicht“" werden bzw. indem ihre Anliegen zu weiblichen Anliegen gemacht werden. Ähnliches soll wohl mit der Aussage von Kurt Möller suggeriert werden: dass es paradox sei, wenn Jungen eine feministische Haltung einnehmen. ${ }^{19}$

Männlichkeitskritik verdankt feministischen Theorien und Praxen entscheidende Impulse. Gemeinsam entwickeln sie Strategien für die Durchsetzung einer umfassenden Geschlechterdemokratie - und folgen dabei selbst einem Demokratiekonzept, das Chantal Mouffe (vgl. 1997) formuliert hat:

19 Frauen, die sich für Frauenrechte einsetzten, und Männer, die Frauen darin unterstützten, wurden um die Jahrhundertwende abwertend „Feministen“ genannt. Ich zitiere aus dem Artikel Die Feministen, den der Freud-Schüler Fritz Wittels 1908 in der Fackel publiziert hat: „Ein sehr großer Teil der Feministen besteht aus Spießbürgern, die auf ein fortschrittliches Programm en bloc eingeschworen sind. Sie meinen, dass man alles emanzipieren müsse: die Juden, die Neger und also auch die Frauen.“ (12) Und an anderer Stelle: „Die unerbittlichsten Feministen endlich sind die, deren Liebe einer emanzipierten Frau gehört.“ (13) (Ich danke Andrea Bramberger für diesen Hinweis.) 
Männlichkeitskritik benötigt keine Grenzen zum Feminismus, keine „eigenen" Strategien, keine Selbständigkeit und Unabhängigkeit, sondern die Bereitschaft, sich auf ein Projekt einzulassen. Die ,wenigen' Grundregeln, die die Voraussetzung für (Geschlechter-)Demokratie bilden, betreffen nichts als die Bereitschaft, in der Verhandlung mit anderen die eigene Identität zu verändern. Die Grundlage für einen solchen Verhandlungsprozess ist freilich, dass diese Bereitschaft von allen gleichermaßen gegeben ist. Und das ist die Grundlage dafür, dass alle an der Verhandlung teilnehmen können, denn Exklusionen aus einem als offen konzipierten Projekt beruhen immer darauf, dass die Nichtausgeschlossenen ihre Identität (ihre Privilegien) eben nicht aufgeben wollen und damit aus einem zukünftigen Projekt eine gegenwärtige Einheit machen.

\section{Wider Resouveränisierung und Immunisierung}

Pierre Bourdieu beginnt seine Ausführungen über Die männliche Herrschaft mit einer erkenntnistheoretischen Betrachtung. Er verweist nicht nur darauf, dass Wissenschafter mit Vorurteilen und Vorannahmen an ihre Gegenstände herangehen, sondern dass sie Wahrnehmungs- und Denkkategorien als Erkenntnismittel verwenden, die sie als Erkenntnisgegenstände analysieren möchten (vgl. Bourdieu 1997a, 153ff.). Die Schwierigkeiten der Sozialwissenschaften bestehen darin, Instrumente zum Denken zu verwenden, die bereits konstruiert sind. „Alles, worüber wir zum Denken verfügen, ist bereits gendered, geschlechtsstrukturiert“ (Bourdieu 1997b, 221). Es geht aber nicht allein darum, die Erkenntnismittel auf ihre impliziten Voraussetzungen zu kontrollieren, denn das imaginiert noch einmal die Illusion, dass über die Kontrolle all der Vorannahmen eine ,reine' Analyse möglich sei, während jede Form des Theoretisierens unabhängig von den verwendeten Denk- und Wahrnehmungskategorien den Gegenstand der Erkenntnis im Sinne der Theorie der Performativität reinszeniert.

Ich habe an anderer Stelle zu zeigen versucht (vgl. Forster 2004), auf welche Weise die Unterschlagung des performativen Effekts in Robert Connells Entwurf einer akademischen Disziplin Kritische Männerforschung wirksam wird und die kritische Intention in ihr Gegenteil umschlägt. Die kritische ,Rede' von hegemonialer Männlichkeit und Patriarchatsdividende erzeugt nämlich einen eigentümlichen Effekt: Sie wird suspekt, weil sie mit der kritischen Position auch ein ,starkes', unangreifbares Subjekt erzeugt. Kritik er- 
scheint, so meine These, als Immunisierungsstrategie gegen die Möglichkeit von Kritik und als Resouveränisierungsstrategie des männlichen Subjekts. ${ }^{20}$

Mir geht es nicht darum, Connells kritisches Männlichkeitsprojekt einfach $\mathrm{zu}$ verwerfen. Es geht auch nicht um andere Fragen oder eine andere Theorie, mit der Männlichkeitsforschung angemessener betrieben werden könnte. Ich möchte vielmehr auf einen kaum wahrgenommenen Punkt insistieren, an dem Kritik kippt und sich in ihr Gegenteil verkehrt. Dieses Kippmoment entsteht durch Connells Strategie der Exklusion. Sie erzeugt ein mächtiges, wissendes Subjekt, das die Definitionsmacht über einen Gegenstand an sich gerissen hat. Dadurch bringt Connell eine fatale Koinzindenz hervor: Er inthronisiert sich als eben jenes männliche, wissende Subjekt, das er auf der Objektseite kritisiert. Weshalb taucht diese Reproduktion einer klassischen männlichen Position bei Connell nicht als Widerspruch auf? Connell ist seine eigene Rede nicht suspekt, weil er sie nicht als (seine) ,Rede', sondern als eine von ihm abgekoppelte Deskription über ,Wirklichkeit" begreift. Mit anderen Worten: Er negiert die Performativität seines Textes, läßt sich als Autor-Ich verschwinden und verwandelt seine ,Rede' in eine Reihe konstativer Statements. Auf diese Weise entsteht eine kritische Männlichkeitsforschung im Rahmen eines Wissenschaftsverständnisses, das von feministischen Theoretikerinnen (vgl. z.B. Fox-Keller 1998, Haraway 1995, Harding 1998; 1994) immer wieder als männlich und/oder phallogozentrisch kritisiert worden ist.

Rituale der Kritik, der vernünftigen Rede und einer abstrakten Selbstreflexion zielen auf die Reproduktion eines - kritischen - Subjekts des Diskurses über Männerforschung. Sie alle machen das Subjekt unangreifbar. Wenn für hegemoniale Männlichkeit ihre Unsichtbarkeit charakteristisch ist, so wird diese von kritischer Männerforschung ans Licht gezerrt - um den Preis, dass sich nur der blinde Fleck verschiebt und nun in der Theorieproduktion wirksam ist.

20 Vgl. dazu auch die kritische Sammelrezension von Siegfried Kaltenecker (1998, 125): „Hier werden allerorts große Programme geschmiedet, feministische Defizite fokussiert und die Unumgänglichkeit eines männerspezifischen Blicks eingeklagt. Selbsternannte Kritiker gerieren sich als ,Saboteure' der Männerherrschaft, um gleichzeitig auf den ,eigenen Interessen als Männer` zu beharren und ,zuerst einmal für mich (zu) kämpfen‘.“ 


\section{Politische Strategien}

Dies verlangt nach einer doppelten politischen Strategie, die zunächst in einem kritischen Gestus besteht, der nicht zu einer Resouveränisierung oder Immunisierung von Männlichkeit führt und ein starkes männliches Subjekt des Genderdiskurses (re-)produziert. In der Praxis lassen sich Resouveränisierungs- oder Immunisierungsstrategien sehr leicht daran erkennen, wie Männerforschung und Männerarbeit ihr Verhältnis zu feministischen Theorien und Praxen definieren. Was heißt das praktisch?

○ Männlichkeitskritik übt radikale Wissenschaftskritik derart, wie etwa Haraway sie formuliert.

○ Männlichkeitskritik formiert keine neue Disziplin (vgl. dagegen Connell 2000, Forster 2004).

○ Keine Lehrstühle für Gender Studies an Männer; Männlichkeitskritik ist eine Praxis, die Männer von dort aus machen, wo sie immer schon sitzen.

○ Männlichkeitskritik heißt für MännerVerantwortung zu übernehmen für eine demokratische Geschlechterpolitik.

○ Männlichkeitskritik heißt: Sich einzumischen - wie? wozu?

Zum anderen müsste die politische Strategie lauten, offene Räume zu schaffen, die dazu animieren, in Verhandlungen zu treten oder, wie Donna Haraway (1990) es ausdrückt, ,partial connections“ zu bilden. In Verhandlungen $\mathrm{zu}$ treten heißt, mit anderen eine „Gruppe“ zu bilden. Gruppen werden nicht durch gegebene Gemeinsamkeiten, durch gegebene Identitäten bestimmt, sondern durch die Bereitschaft, solche zu (er)finden. Der Ausgangspunkt ist nicht das Gegebene, sondern eine (mögliche) Zukunft. Elspeth Probyns (1995) Begriff der „queer belongings“ bezeichnet diese Haltung: Sich nicht auf vorgegebene, hegemoniale Zugehörigkeiten zu verlassen, sondern in einen aktiven Prozess des (Er-)Findens einzutreten. Beziehungen dieser Qualität sind offen, kontextgebunden, flüchtig. Solche Formen der Gruppenkonstituierung zu verallgemeinern heißt, politische Gemeinschaften zu entwerfen, die sich nicht auf ein Geschlecht, eine Nation, eine Kultur, eine Ethnie, eine Generation berufen, sondern auf eine unabsehbare Zukunft. 


\section{Literatur}

Althusser, Louis (1977): Ideologie und ideologische Staatsapparate. Aufsätze zur marxistischen Theorie. Hamburg/Westberlin

BauSteineMänner (Hg.) (2001): Kritische Männerforschung. Neue Ansätze in der Geschlechtertheorie. Hamburg: Argument (3., erweiterte Aufl.)

Becker-Schmidt, Regina (1993): Geschlechterdifferenz - Geschlechterverhältnis: Soziale Dimensionen des Begriffs „Geschlecht“. In: Zeitschrift für Frauenforschung 11 , Heft $1 / 2$

Bly, Robert (1991): Eisenhans. Ein Buch über Männer, München

Brandes, Holger (2002): Der männliche Habitus. Band 2: Männerforschung und Männerpolitik, Opladen

Bieringer, Ingo/Buchacher, Walter/Forster, Edgar (Hg.) (2000): Männlichkeit und Gewalt. Konzepte für die Jungenarbeit, Opladen

Böhnisch, Lothar (2001): Männlichkeiten und Geschlechterbeziehungen - Ein männertheoretischer Durchgang. In: Margrit Brückner \& Lothar Böhnisch (Hg.): Geschlechterverhältnisse. Gesellschaftliche Konstruktionen und Perspektiven ihrer Veränderung, Weinheim \& München

Bohnsack, Ralf (2001): Der Habitus der „Ehre des Mannes“. Geschlechtsspezifische Erfahrungsräume bei Jugendlichen türkischer Herkunft. In: Döge, Peter \& Meuser, Michael (Hg.): Männlichkeit und soziale Ordnung. Neuere Beiträge zur Geschlechterforschung, Opladen

Bourdieu, Pierre (1997a): Die männliche Herrschaft. In: Dölling, Irene \& Krais, Beate (Hg.): Ein alltägliches Spiel. Geschlechterkonstruktion in der sozialen Praxis, Frankfurt a.M.

Bourdieu, Pierre (1997b): Eine sanfte Gewalt. Pierre Bourdieu im Gespräch mit Irene Dölling und Margareta Steinrücke. In: Dölling, Irene \& Krais, Beate (Hg.): Ein alltägliches Spiel. Geschlechterkonstruktion in der sozialen Praxis, Frankfurt a.M.

Butler, Judith (1991): Das Unbehagen der Geschlechter, Frankfurt a.M.

Connell, Robert W. (2000): The Men and the Boys. Berkeley \& Los Angeles: UC Press

Connell, Robert W. (1999): Der gemachte Mann. Konstruktion und Krise von Männlichkeiten, Opladen

Cornell, Drucilla (1991): Beyond Accomodation. Ethical Feminism, Deconstruction, and the Law. Lanham u.a.: Rowman \& Littlefield (New Edition).

Copjec, Joan (1995): Das Geschlecht und die Euthanasie der Vernunft. In: texte psychoanalyse. ästhetik. kulturkritik 15, H. 1

Deleuze, Gilles (1997): David Hume, Frankfurt a.M.

Deleuze, Gilles (1993): Logik des Sinns, Frankfurt a.M.

Deleuze, Gilles (1989): Zeichen und Ereignisse. Ein Gespräch mit François Ewald und Raymond Bellour. In: Pariser Gespräche, Berlin 
Deleuze, Gilles/Guattari, Félix (1997): Tausend Plateaus. Kapitalismus und Schizophrenie, Berlin

Derrida, J. (1998): Auslassungspunkte - Gespräche, Wien

Derrida, Jacques (1993): Falschgeld - Zeit geben I, München

Derrida, Jacques (1986): Positionen. Gespräche mit Henri Ronse, Julia Kristeva, JeanLouis Houdebine, Guy Scarpetta, Graz/Wien

Döge, Peter (2001): Geschlechterdemokratie als Männlichkeitskritik. Blockaden und Perspektiven einer Neugestaltung des Geschlechterverhältnisses, Bielefeld

Döge, Peter/Meuser, Michael (2001): Männlichkeit und soziale Ordnung. Neuere Beiträge zur Geschlechterforschung, Opladen

Douglas, Mary (1991): Wie Institutionen denken, Frankfurt a.M.

Duden, Barbara (1993): Die Frau ohne Unterleib: Zu Judith Butlers Entkörperung. Ein Zeitdokument. In: Feministische Studien, H. 2

Düttmann, A. G. (1992): Die Dehnbarkeit der Begriffe. Über Dekonstruktion, Kritik und Politik. In: Georg-Lauer, J. (Hg.): Postmoderne und Politik, Tübingen

Engelmann, Peter (1986): Positionen, 14 Jahre später. In: Derrida, Jacques: Positionen. Gespräche mit Henri Ronse, Julia Kristeva, Jean-Louis Houdebine, Guy Scarpetta, Graz/Wien

Falk, Gunter/Steinert, Heinz (1973): Über den Soziologen als Konstrukteur von Wirklichkeit, das Wesen der sozialen Realität, die Definition sozialer Situationen und die Strategien ihrer Bewältigung. In: Steinert, Heinz (Hg.): Symbolische Interaktion. Arbeiten zu einer reflexiven Soziologie, Stuttgart

Forster, Edgar (2004): Männlichkeitsrituale als Widerstandsrituale in Erziehung und Bildung. Zur Konstruktion des (kritischen) Subjekts im Männlichkeitsdiskurs. In: Zeitschrift für Erziehungswissenschaft, 6. Jg., Beiheft 2

Forster, Edgar (2002): Jungenarbeit als Männlichkeitskritik. In: Kofra - Zeitschrift für Feminismus und Arbeit, Heft 96

Forster, Edgar (2001): Die Beweglichkeit des Rassismus. In: TANGRAM - Bulletin der Eidgenössischen Kommission gegen Rassismus. Nr. 10

Forster, Edgar/Rieger-Ladich, Markus (2004): Männerforschung und Erziehungswissenschaft. In: Glaser, Edith/Klika, Dorle/Prengel, Annedore (Hg.): Handbuch Gender und Erziehungswissenschaft, Bad Heilbrunn

Foucault, Michel (1996): Der Mensch ist ein Erfahrungstier, Frankfurt a.M.

Fox-Keller, Evelyn (1998): Liebe, Macht und Erkenntnis. Männliche oder weibliche Wissenschaft? Frankfurt a.M.

Fraser, Nancy (2001): Die halbierte Gerechtigkeit. Schlüsselbegriffe des postindustriellen Sozialstaats, Frankfurt a.M.

Grossberg, Lawrence (2000): What's going on? Cultural Studies und Popularkultur, Wien

Grossberg, Lawrence (1996a): Identity and Cultural Studies - Is That All There Is? In: Stuart Hall and Paul duGay (Eds), Questions of Cultural Identity. London/Thousand Oaks/New Delhi 
Grossberg, Lawrence (1996b): History, Politics and Postmodernism. In: David Morley, Kuan-Hsing Chen (eds.): Stuart Hall. Critical Dialogues in Cultural Studies. London/New York: Routledge

Hall, Stuart (2000): Cultural Studies. Ein politisches Theorieprojekt. Ausgewählte Schriften 3, Hamburg

Hall, Stuart (1996): Introduction: Who Needs 'Identity'? In: Hall, Stuart/Paul du Gay (eds.): Questions of Cultural Identity. London u. a.

Haraway, Donna (1995): Situiertes Wissen. Die Wissenschaftsfrage im Feminismus und das Privileg einer partialen Perspektive. In: Dies.: Die Neuerfindung der Natur. Primaten, Cyborgs und Frauen. Frankfurt/New York

Haraway, Donna (1990): A Manifesto for Cyborgs: Science, Technology, and Socialist Feminism in the 1980s. In: Nicholson, Linda J.: Feminism/Postmodernism. New York/London

Harding, Sandra (1998): Is Science Multicultural? Postcolonialisms, Feminisms, and Epistemologies. Bloomington/Indianapolis

Harding, Sandra (1994): Das Geschlecht des Wissens. Frauen denken die Wissenschaft neu, Frankfurt/New York

Haywood, Chris/Mac an Ghaill, Máirtín (2003): Men and Masculinities - Theory, research and social practice. Buckingham-Philadelphia.

Kafka, Franz (1994): Tagebücher. Band 3: 1914-1923 (in der Fassung der Handschrift). Frankfurt a.M.

Kaltenecker, Siegfried (1998): Schwindelnde Männer. Neues zum Thema Männlichkeit. In: Widersprüche - Zeitschrift für sozialistische Politik im Bildungs-, Gesundheits- und Sozialbereich, Heft 67 (18. Jg.), Nr. 1

Knapp, Gudrun-Axeli (1992): Macht und Geschlecht. Neuere Entwicklungen in der feministischen Macht und Herrschaftsdiskussion. In: Gudrun-Axeli Knapp und Angelika Wetterer (Hg.): TraditionenBrüche. Entwicklungen feministischer Theorie, Freiburg

Laclau, Ernesto (1997): Inklusion, Exklusion und die Logik der Äquivalenz (Über das Funktionieren ideologischer Schließungen). In: Weibel, Peter/Žižek, Slavoj (Hg.): Inklusion : Exklusion. Probleme des Postkolonialismus und der globalen Migration, Wien

Meuser, Michael (1998): Geschlecht und Männlichkeit. Soziologische Theorie und kulturelle Deutungsmuster, Opladen

Meuser, Michael \& Behnke, Cornelia (1998): Tausendundeine Männlichkeit? Männlichkeitsmuster und sozialstrukturelle Einbindungen. In: Widersprüche - Zeitschrift für sozialistische Politik im Bildungs-, Gesundheits- und Sozialbereich, Heft 67 (18. Jg.), Nr. 1

Möller, Kurt (1997): Zur Einführung: Merkmale und Konturen sozialer und pädagogischer Arbeit am Männlichkeits-Spagat. In: Ders. (Hg.): Nur Macher und Macho? Geschlechtsreflektierende Jungen- und Männerarbeit, Weinheim/München 
Mouffe, Chantal (1997): Inklusion/Exklusion: Das Paradox der Demokratie. In: Weibel, Peter/Žižek, Slavoj (Hg.): Probleme des Postkolonialismus und der globalen Migration, Wien

Probyn, Elsbeth (1995): Queer Belongings. Eine Politik des Aufbruchs. In: Angerer, Marie-Luise (Hg.): The Body of Gender. Körper, Geschlechter, Identitäten, Wien

Straub, Jürgen: Personale und kollektive Identität. Zur Analyse eines theoretischen Begriffs. In: Assmann, Aleida \& Friese, Heidrun (Hg.): Identitäten - Erinnerung, Geschichte, Identität 3. Frankfurt a.M.

Stuve, Olaf (2001): „Queer Theory“ und Jungenarbeit. Versuch einer paradoxen Verbindung. In: Fritzsche, Bettina/Hartmann, Jutta/Schmidt, Andrea/Tervooren, Anja (Hg.): Dekonstruktive Pädagogik. Erziehungswissenschaftliche Debatten unter poststrukturalistischen Perspektiven, Opladen

Tillner, Georg/Kaltenecker, Siegfried (1995): Offensichtlich männlich. Zur aktuellen Kritik der heterosexuellen Männlichkeit. In: Texte zur Kunst, 5. Jg., Nr. 17

Walter, Willi (2000): Gender, Geschlecht und Männerforschung. In: Braun, Christina von \& Stephan, Inge (Hg.): Gender-Studien. Eine Einführung, Stuttgart \& Weimar

Whitehead, Stephen M. (2002): Men and Masculinities. Key Themes and New Directions. Cambridge: Polity

Wittels, Fritz: Die Feministen. In: Die Fackel, Nr. 248, 24. März 1908

Young, Iris Marion (1994): Geschlecht als serielle Kollektivität: Frauen als soziales Kollektiv. In: Geschlechterverhältnisse und Politik. Hg. vom Institut für Sozialforschung Frankfurt (Redaktion: Katharina Pühl). Frankfurt a.M.

Žižek, Slavoj (1994): The Sublime Object of Ideology. London/New York 